\title{
Pendulum as a model system for driven rotation in molecular nanoscale machines
}

\author{
A. V. Zolotaryuk, ${ }^{1,2}$ P. L. Christiansen, ${ }^{1}$ B. Nordén, ${ }^{3}$ A. V. Savin, ${ }^{1,4}$ and Y. Zolotaryuk ${ }^{1, *}$ \\ ${ }^{1}$ Department of Mathematical Modelling, Technical University of Denmark, DK-2800 Lyngby, Denmark \\ ${ }^{2}$ Bogolyubov Institute for Theoretical Physics, 03143 Kyiv, Ukraine \\ ${ }^{3}$ Department of Physical Chemistry, Chalmers University of Technology, S-412 96 Gothenburg, Sweden \\ ${ }^{4}$ Institute for Problems of Physics and Technology, 119034 Moscow, Russia
}

(Received 8 July 1999)

\begin{abstract}
We suggest a ratchet mechanism of rotatory (or translatory) motion of a Brownian rotator (or a particle) in a spatially symmetric periodic potential. The asymmetry that drives the ratchet motion is due to a special sequence of activation of catalytic sites arranged in space circularly and periodically. A pendulum driven by short impulses at its stable equilibrium point is shown to be a simple mechanical model which can be constructed easily and used for visual observation of the ratchet rotation. A possible application of this mechanism in nanotechnology is briefly discussed.
\end{abstract}

PACS number(s): 87.15.-v, 82.20.Fd, 05.40.-a, 07.10.Cm

In order to power nanoscale devices, like twirling microscopic plastic beads, now researchers are turning to biology and have already undertaken the first steps in singling out protein-based motors from living systems and remounting them on engineered surfaces to perform usable work [1]. One of these is $F_{1}$-ATPase (or the $F_{1}$ motor), the portion of the ATP synthase which is exposed on the cell membrane surface. This is the smallest motor in the world and its rotational motion was recently observed by Noji et al. [2]. The $\mathrm{F}_{1}$ motor is remarkably sophisticated, containing a cylinder of six subunits with three catalytic sites (stator) and surrounding a central shaft (rotor), the so-called $\gamma$ subunit. As for any biological motor, adenosine triphosphate (ATP) is chemical fuel, making the unidirectional rotation. On the other hand, molecular dynamics approach using rotational impulse dynamics has been developed recently for studying the molecular nanomachine designs [3]. Various power input profiles to drive devices such as nanogears and pumps have been used in these studies with atomistic details. Therefore we may expect that nanostructures with high-frequency rotational motions can be manufactured using some principles of functioning $F_{1}$-ATPase. In particular, the so-called binding change mechanism proposed by Boyer and Walker on biochemical grounds $[4,5]$ could be explored for these purposes.

In this paper we report a ratchet mechanism of the unidirectional rotational motion which can be used for possible manufacturing devices at the nanoscale level. Inspired by Feynman's thermal ratchet [6], a variety of mechanisms for molecular motors have been suggested [7]. All involve rectifying motion of a Brownian particle in a spatially periodic structure due to zero-mean nonequilibrium oscillations or fluctuations. The fundamental condition for such a rectified transport to occur is that either the spatial reflection symmetry of the system is broken [8-13] or fluctuations are statistically asymmetric [14-18]. As a simple mechanical model,

\footnotetext{
*Author to whom correspondence should be addressed. Present address: Max-Planck-Institut für Physik komplexer Systeme, Nöthnitzer Str. 38, 01187, Dresden, Germany. Electronic address: yzolo@mpipks-dresden.mpg.de
}

here we consider the pendulum, biasing rotation of which is generated by a special kind of temporal asymmetry that differs from those previously reported [14-18].

Let us consider an underdamped pendulum (or the same, a particle subjected to a sinusoidal potential) of mass $m$ and length $l$ which is subject to white noise $\xi(t)$ of zero average. We assume that the pendulum is also driven by an external torque $F(t)$ which depends on the angle position $\theta(t)$ as well as on the velocity $\dot{\theta}(t)$. The time evolution of $\theta(t)$ is governed by the equation of motion

$$
m l^{2} \ddot{\theta}+\gamma \dot{\theta}+m g \sin \theta=F(t)+\xi(t),
$$

where $\gamma$ is a friction constant and $g$ the gravitational acceleration. The driving torque $F(t)$ is assumed to be generated by short impulses of the same amplitude applied upwards only at those time instants when the pendulum, after it has turned some integer number of total revolutions, either counterclockwise (the positive direction of $\theta$ ) or clockwise (the negative direction of $\theta$ ), is passing the lowest (stable equilibrium) point. Then the pendulum will be affected tangentially by a clockwise or counterclockwise torque $F(t)$ with probability $1 / 2$, so that $\langle F(t)\rangle=0$. Let $\left\{t_{j}\right\}_{j=0}^{\infty}$ be the sequence of these time instants. For each $j$ we introduce the two integers $n_{j}^{ \pm}$that determine the number of positive $\left(n_{j}^{+}\right)$ or negative $\left(n_{j}^{-}\right)$revolutions which are required to occur after the kick at $t=t_{j}$ in order to receive the next kick at $t$ $=t_{j+1}$. For instance, the pendulum can be supposed to receive the energy after it has turned counterclockwise each time $\left(n_{j}^{+}=1\right)$ or clockwise two times $\left(n_{j}^{-}=-2\right)$ as depicted in Fig. 1. Experimentally, such a forcing system could be constructed by using some electric circuit control devices, including photocells, electromagnets, etc., that switch a $\delta$-like force directed upwards at those time instants when the pendulum is passing the lowest point after a corresponding number $n_{j}^{ \pm}$of revolutions. The white noise approximately can be created by a fog-horn applied perpendicularly to the plane in which the pendulum rotates. Correspondingly, in chemical terms, a Brownian particle thermally fluctuating in a periodic potential shown in Fig. 1 can be activated selec- 


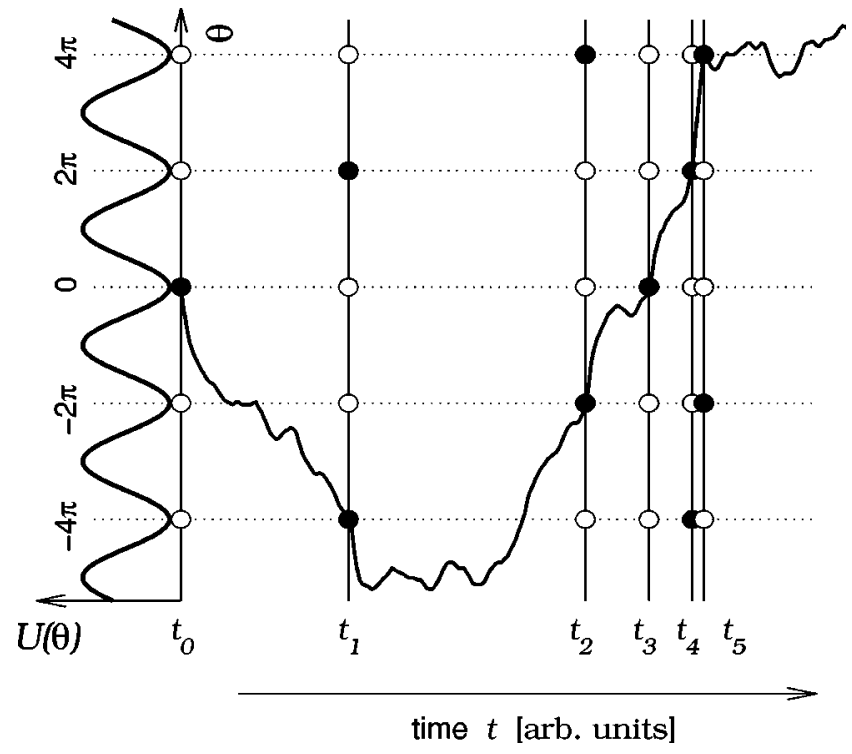

FIG. 1. The dimensionless potential $U(\theta)=1-\cos \theta$ as a function of the angle position $\theta$ (displayed vertically) and one of the realizations of the stochastic process $\theta(t)$ with a time random sequence $t_{0}, t_{1}, \ldots$. Initially, at $t=t_{0}$ the pendulum was occasionally pushed backwards, but afterwards its global motion appeared to occur forward. The random trajectory is shown to pass through closed circles that correspond to the active states. The passive states are represented by open circles.

tively, while being at some minima, to overcome the potential barrier to the nearest-neighbor potential well, either forward or backwards with the same probability $1 / 2$.

Let $W$ be a kinetic energy given to the pendulum during each impulse stroke. Because of this energy input, the angular velocity of the pendulum being initially at rest is increased by $\Delta \omega=l^{-1} \sqrt{2 W / m}$, so that from Newton's law $F \Delta t=m l^{2} \Delta \omega(\Delta t$ is duration of the impulse) one obtains in the limit $1 / \Delta t \rightarrow \delta(t)$ the torque amplitude $l \sqrt{2 m W}$. Therefore $t_{0}=l \sqrt{m / 2 W}$ can be chosen as a time unit and the dimensionless form of the dynamical equation (1) takes the form

$$
\frac{d^{2} \theta}{d \tau^{2}}+\Gamma \frac{d \theta}{d \tau}=-\varepsilon_{0} \sin \theta+\sum_{j=0}^{\infty}( \pm)_{j} p_{j}^{ \pm} \delta\left(\tau-\tau_{j}\right)+\eta(\tau)
$$

with $\tau=t / t_{0}, \Gamma=\sqrt{I / 2 W} \gamma$, and $\varepsilon_{0}=E_{0} / 2 W$. The correlation function of the white noise $\eta(\tau)=\xi(t) / 2 W$ is $\left\langle\eta(\tau) \eta\left(\tau^{\prime}\right)\right\rangle$ $=2 \Gamma D \delta\left(\tau-\tau^{\prime}\right)$ with strength $D=k_{B} T / 2 W\left(k_{B}\right.$ is Boltzmann's constant and $T$ temperature). For nanotechnological applications the white noise models thermal fluctuations (at room temperature $k_{B} T \simeq 4 \mathrm{pN} \mathrm{nm}$ ). The coefficient $p_{j}^{ \pm}$describes the probability with which at the time $\tau=\tau_{j}$ the pendulum is kicked forward $\left(p_{j}^{+}\right)$or back $\left(p_{j}^{-}=1-p_{j}^{+}\right)$. As described above, $p_{j}^{+}=p_{j}^{-}=1 / 2$. The symbol $( \pm)_{j}$ means 1 or -1 , respectively.

It is reasonable to assume that each probability $p_{j}$ in Eq. (2) depends on the velocity $\Omega_{j}=\Omega\left(\tau_{j}\right)$ given at $\tau=\tau_{j}$ where $\Omega=d \theta / d \tau$. Indeed, while bypassing the lowest point, under the same stochastic action as described above the pendulum receives each stroke with some finite delay depending on the velocity $\Omega$ at this time instant. Clearly, the maximal change of this dependence takes place if $p_{j}^{+}=p_{j}^{-}=1 / 2$. Also, any

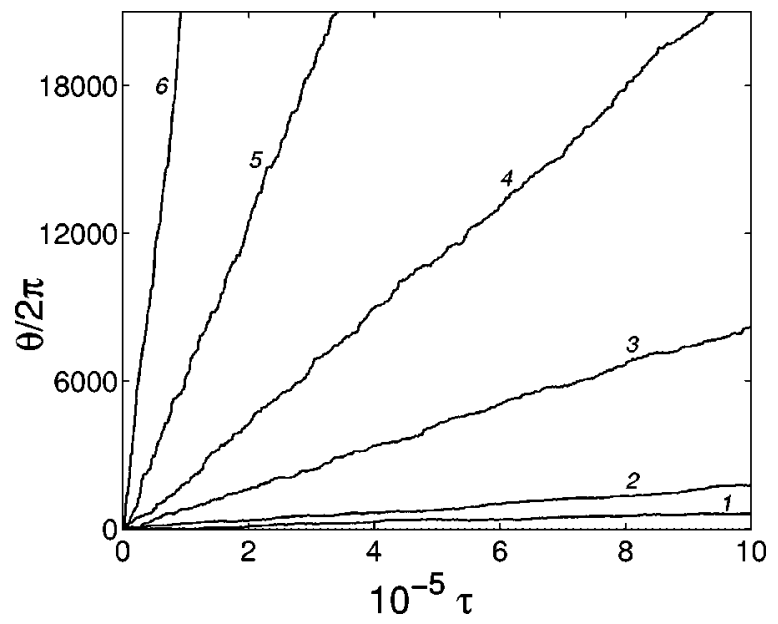

FIG. 2. Molecular dynamics simulation of the pendulum rotation $\left(\Gamma=0.5\right.$ and $\varepsilon_{0}=0.05$ ) for $\mu=0$ (curve 1 ), $\mu=1$ (curve 2), $\mu=3$ (curve 3), $\mu=5$ (curve 4 ), $\mu=10$ (curve 5), and $\mu=100$ (curve 6).

equation must be invariant with respect to the substitution of $p_{j}^{+}$by $1-p_{j}^{-}$. Using these two general assumptions, one can write the following nonlinear differential equation

$$
\frac{d p_{j}^{ \pm}}{d \Omega_{j}}= \pm \mu\left(1-p_{j}^{ \pm}\right) p_{j}^{ \pm}
$$

where $\mu$ is a positive coefficient that determines the time delay of the pendulum-source interaction. Equation (3) can be easily solved and its solution reads

$$
p_{j}^{ \pm}=\frac{1}{2}\left(1 \pm \tanh \frac{\mu \Omega_{j}}{2}\right) .
$$

The only possibility to drive unidirectionally the pendulum with a forcing of zero average as described above is to introduce some kind of temporal asymmetry in the sequence of impulses generating the stochastic torque $F(t)$. We impose this asymmetry by assuming sequences $n_{j}^{+}$and $n_{j}^{-}$to be different. Despite the torque $F(t)$ is mean centered, such a difference is expected to cause a unidirectional rotation of the pendulum. We emphasize that both possibilities $p^{+}$ $>p^{-}$or $p^{+}<p^{-}$may happen and they are equally considered. Intuitively, the ratchet is expected to occur in the counter clockwise (positive) direction if some strokes are missed in a regular or random manner after the pendulum has turned clockwise. We report here the particular case when for each $j$ the pendulum receives the energy $W$ each first-passage time forward $\left(n_{j}^{+}=1\right)$, but each second-passage time backwards $\left(n_{j}^{-}=-2\right)$, the case mentioned above. In this case, at the time instant $t_{j}$ the pendulum can perform any number of revolutions from the sequence $3 i-2 j, i$ $=0,1, \ldots, j$.

The numerical integration of Eq. (2) has shown that the rectification of the rotation indeed occurs for all values of $\mu \geqslant 0$ (see Fig. 2). These results clearly demonstrate a high increase of the rectification efficiency with $\mu$. Two of these realizations for small periods of integration and small values of $\mu$ are shown separately in Fig. 3. Thermal fluctuations nearby the potential minima are demonstrated by Fig. 3(a) 

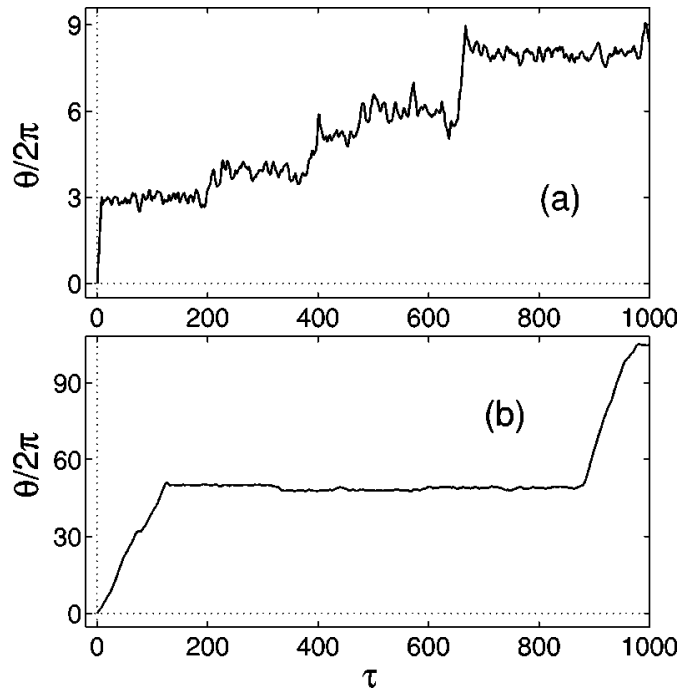

FIG. 3. Curves 2 and 5 of Fig. 2 displayed for small period of integration.

whereas Fig. 3(b) shows the existence of a temporal plateau describing the stop of the ratchet mechanism at some angular position of the pendulum at the lowest point, which remarkably resembles the experimental observation of the $\gamma$-subunit rotation in $F_{1}$-ATPase [19]. Numerical calculations of the averaged velocity against the barrier height $\varepsilon_{0}$ (see Fig. 4) have shown that there exists a certain optimal value of the barrier height at which the ratchet occurs with the highest efficiency. The global rotation disappears with increase of impulse strength. However, for $\mu>0$ the ratchet still occurs for larger amplitudes of impulses.

As an application of the described mechanism for driven rotation in nanostructures, let us consider a finite lattice consisting at least of three catalytic sites arranged circularly and periodically. Let $N$ be a number of these sites denoted by $1, \ldots, N, N \geqslant 3$, in the positive direction which form a stator. We assume that this array of sites surrounds a rotating

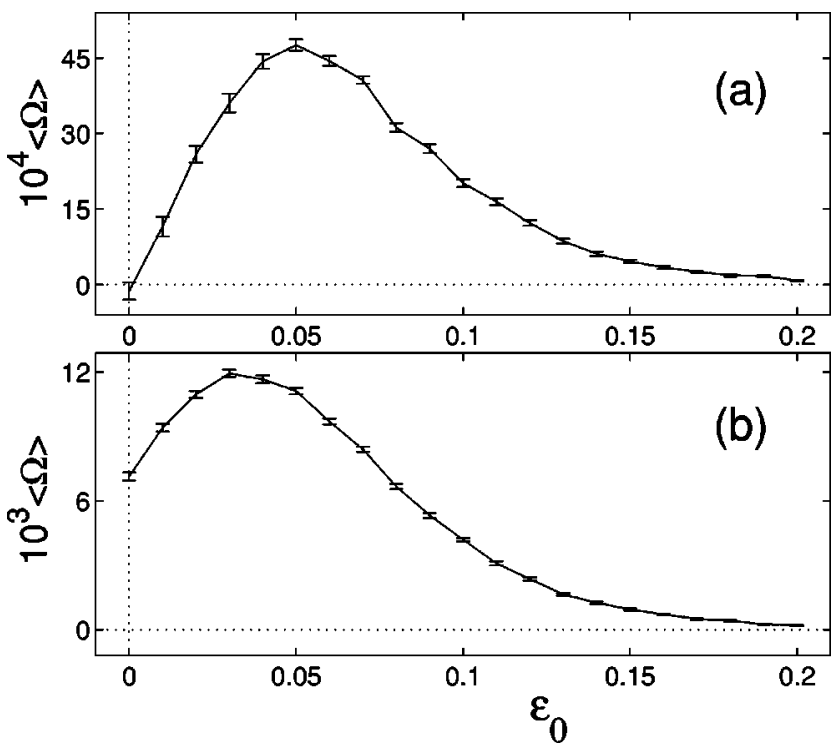

FIG. 4. Averaged global angular velocity of the pendulum rotation against the dimensionless potential barrier height for (a) $\mu$ $=0$ and (b) $\mu=1$. shaft, forming a periodic potential $U(\theta)$ with the period $2 \pi / N$. Assume next that each of the stator sites can be either active or passive with respect to a certain chemical site on the rotor surface, but only one of the $N$ sites can be active at a time. When the active site at the rotor is getting the closest to the stator site found in the active state, i.e., when the rotor reaches the state due to thermal fluctuations, it receives a power stroke (impulse) during the chemical reaction at this state. For instance, this may be the hydrolysis of an ATP molecule with $W \simeq 80 \mathrm{pN} \mathrm{nm}$ being the released hydrolysis energy. After the reaction has happened to occur at the active site of the stator, the next site in the positive direction becomes active, so that the rotor can reach it turning (due to thermal fluctuations and the hydrolysis impulse) either forward by $2 \pi / N$ or backwards by $-2 \pi(N-1) / N$. This system is equivalent to the pendulum being kicked at the lowest point as described above. The only difference is due to scaling of the period of the function $U(\theta)$ : instead of $2 \pi$, here we have $2 \pi / N$. This ratchet effect can be termed a trapping of a particle by a moving sequence of active catalytic states along a cyclic lattice.

Now the next question arises: how such a "moving" sequence of active catalytic states can be arranged. To answer this question, one of the ways is to turn to the above mentioned $F_{1}$-ATPase enzyme, an extremely interesting example of nanoscale machine in living things, which was recently discovered to run, burning ATP molecules to rotate the central $\gamma$-subunit shaft [2]. The stator of this motor is the hexagonal array of three $\alpha$ subunits and three $\beta$ subunits arranged alternatingly. According to the binding change mechanism $[4,5]$, three potentially catalytic nucleotidebinding sites which are located on the $\beta$ subunits can be found sequentially in one of the three conformational states: ATP binding $\left(\beta_{T}\right)$, ADP binding $\left(\beta_{D}\right)$, and empty $\left(\beta_{E}\right)$. At each rotation by $2 \pi / 3$, one ATP molecule is bound to the $\beta_{E}$ state, while one ADP molecule and phosphate are released from the $\beta_{D}$ state, resulting in the following sequence of conformational changes: $\beta_{E} \rightarrow \beta_{T}, \beta_{T} \rightarrow \beta_{D}$, and $\beta_{D} \rightarrow \beta_{E}$. This $2 \pi / 3$ rotation drives the $\gamma$-subunit unidirectionally.

Thus, the ratchet mechanism described by the driven and underdamped pendulum equation (2) can be highly relevant for manufacturing nanoscale machine designs. It is mainly based on the central idea of the rotational catalysis, the binding change mechanism in the $F_{1}$-ATPase enzyme. Despite this enzyme is the smallest motor known in the world, it is too complicated system and its mechanism of functioning is far to be fully understood $[2,20,21]$. Therefore the ratchet mechanism suggested in this paper seems to be too simple to describe the work of $F_{1}$-ATPase, but the dynamical model of motion driven by rotational catalysis could be of interest for nanotechnological applications. We emphasize that the unidirectional rotation governed by Eq. (2) with a symmetric potential is a new ratchet model among those containing potentials with broken spatial asymmetry and studied so extensively [7-13]. Another dimension of beauty is the theoretical problem of dynamics and effects of inertia in ratchets $[22,23]$. The rectification with $\mu=0$ is a remarkable phenomenon and this is another case compared with the rectification in a symmetric potential when special types of statistical asymmetry are imposed [14-18]. The stochastic process described above is nonlinear in the sense that the pendulum 
interacts with the driving source, so that the power strokes occur at the times depending on the pendulum position. Such a pendulum-source interaction becomes very effective when the dependence of this interaction (the probabilities $p_{j}^{ \pm}$) on the pendulum velocity is incorporated into the model. This interaction resembles the electron-phonon coupling in solids when an electron "digs" a potential well while this well in turn captures the electron forming a polaron state. The pendulum turning on average more and more frequently in the positive direction causes more frequent kicks. In their turn, the increase of the frequency of kicks results in the growth of biasing the rotational motion, but note that both the probabilities $p_{j}^{+}$and $p_{j}^{-}$are equally "present" in the model and the inequality $p_{j}^{+}>p_{j}^{-}$that occurs more frequently in the process is a result of this "self-trapping" effect. In the limit of large $\mu$, one obtains nearly 100 percent efficient mechanism, so important from the point of view of biological motors.

We thank A. C. Scott for helpful discussions. This research was partially carried out with the financial support from the European Economic Community (EEC) under INTAS Grant No. 97-0368.
[1] R. F. Service, Science 283, 27 (1999).

[2] H. Noji, R. Yasuda, M. Yoshida, and K. Kinoshita, Jr., Nature (London) 386, 299 (1997); H. Noji, Science 282, 1844 (1998).

[3] See, e.g., T. Cagin, A. Jaramillo-Botero, G. Gao, and W. A. Goddard III, Nanotechnology 9, 143 (1998), and references therein.

[4] P. D. Boyer, Biochim. Biophys. Acta 1140, 215 (1993).

[5] J. P. Abrahams, A. G. W. Leslie, R. Lutter, and J. E. Walker, Nature (London) 370, 621 (1994).

[6] R. P. Feynman, R. B. Leighton, and M. Sands, in The Feynman Lectures on Physics, Vol. 1, Ch. 46 (Addison-Wesley, Reading, Massachusetts, 1966).

[7] For a review see F. Jülicher, A. Ajdari, and J. Prost, Rev. Mod. Phys. 69, 1269 (1997); R. D. Astumian, Science 276, 917 (1997).

[8] A. Ajdari and J. Prost, C. R. Seances Acad. Sci., Ser. 2 315, 1635 (1992); J. Rousselet, L. Salome, A. Ajdari, and J. Prost, Nature (London) 370, 446 (1994); J. Prost, J.-F. Chauwin, L. Peliti, and A. Ajdari, Phys. Rev. Lett. 72, 2652 (1994).

[9] M. O. Magnasco, Phys. Rev. Lett. 71, 1477 (1993).

[10] R. D. Astumian and M. Bier, Phys. Rev. Lett. 72, 1766 (1994).

[11] C. R. Doering, W. Horsthemke, and J. Riordan, Phys. Rev. Lett. 72, 2984 (1994).

[12] M.M. Millonas and M.I. Dykman, Phys. Lett. A 185, 65 (1994).
[13] T. E. Dialynas, K. Lindenberg, and G. P. Tsironis, Phys. Rev. E 56, 3976 (1997).

[14] J. Luczka, R. Bartussek, and P. Hänggi, Europhys. Lett. 31, 431 (1995); T. Hondu and Y. Sawada, ibid. 35, 313 (1996); P. Hänggi, R. Bartussek, P. Talkner, and J. Luczka, ibid. 35, 315 (1996).

[15] M. C. Mahato and A. M. Jayannavar, Phys. Lett. A 209, 21 (1995); Phys. Rev. E 55, 3716 (1997); Physica A 248, 138 (1998).

[16] D. R. Chialvo and M. M. Millonas, Phys. Lett. A 209, 26 (1995).

[17] J. Kula, Czernik, and J. Luczka, Phys. Lett. A 214, 14 (1996).

[18] R. Lahiri, e-print cond-mat/9607099.

[19] See Fig. 3 in H. Noji, R. Yasuda, M. Yoshida, and K. Kinosita, Jr., Nature (London) 386, 299 (1997), where several time courses of the $\gamma$-subunit rotation with temporal stoppage are presented.

[20] D. S. Sabbert, S. Engelbrecht, and W. Junge, Proc. Natl. Acad. Sci. USA 94, 4401 (1997).

[21] H. Wang and G. Oster, Nature (London) 396, 279 (1998).

[22] P. Jung, J. G. Kissner, and P. Hänggi, Phys. Rev. Lett. 76, 3436 (1996).

[23] Ya. M. Blanter and M. Büttiker, Phys. Rev. Lett. 81, 4040 (1998). 